\title{
Ethanol administration dampens the prolactin response to psychosocial stress exposure in sons of alcohol-dependent fathers
}

\author{
Ulrich S. Zimmermann ${ }^{\mathrm{a}, *}$, Arlette F. Buchmann ${ }^{\mathrm{b}}$, Constance Spring ${ }^{\mathrm{a}}$, Manfred Uhr ${ }^{\mathrm{a}}$, Florian \\ Holsboer ${ }^{a}$, Hans-Ulrich Wittchen ${ }^{a}$ \\ a Max-Planck-Institute of Psychiatry, Kraepelinstrasse 10, 80804 Munich, Germany \\ b Department of Addictive Behavior and Addiction Medicine, Central Institute of Mental Health, J5, \\ 68159 Mannheim, Germany
}

\section{Summary}

Genetic predisposition and exposure to alcohol and stress increase the risk for alcoholism, possibly by forming a threefold interaction. This is suggested by various aspects of alcohol-induced stress response dampening in offspring of alcoholics. We tested whether such an interaction is also revealed by prolactin secretion, which is predominantly controlled by hypothalamic dopamine. Plasma prolactin was measured during four experimental days in 26 young males with a paternal history of alcoholism (PHA) and in 22 family history negative (FHN) controls. A public speaking stress paradigm was applied on the first 2 days, and a non-stress acoustic startle experiment on the others. Before the tests, subjects drank alcohol $(0.6 \mathrm{~g} / \mathrm{kg})$ or placebo in a randomized, double-blind crossover design. During placebo experiments, prolactin levels significantly increased after stress, but not after startle, and did not differ between risk groups. Alcohol administration significantly increased prolactin before stress and during startle in both groups, did not alter stress-induced prolactin stimulation in FHN, but significantly attenuated the prolactin stress response in PHA subjects. The alcohol effects on prolactin, cortisol, and adrenocorticotropin stress response were positively interrelated with each other. These data confirm that alcohol specifically dampens the stress response in PHA but not FHN subjects. Since prolactin responses to stress alone and alcohol alone were normal in PHA, we conclude that this genetic effect is not related to altered physiology of the hypothalamic dopaminergic system, but to risk-group specific alcohol effects on hierarchically higher brain areas controlling the stress response in general.

Keywords: Stress; Alcoholism; Prolactin; Ethanol; Administration

\section{Introduction}

Offspring of alcohol-dependent parents often develop alcohol use disorders themselves, and more than half of their increased risk can be attributed to genetic factors (Heath et al., 1997; Prescott and Kendler, 1999). In order to find underlying mechanisms, many researchers turned to experimental studies comparing offspring of alcohol-dependent parents with control subjects without alcoholic relatives. In order to distinguish pre-existing alterations from alcohol- induced harm, such studies are preferably performed in young adults who have not yet developed alcohol use disorders themselves. This research strategy revealed anatomical (Hill et al., 2001), electrophysiological (Hada et al., 2001) and endocrine abnormalities (Wand et al., 1998), but the most important findings come from alcohol challenge experiments with these high-risk subjects. For example, the sons of alcoholics were found to show less subjective (Schuckit and Gold, 1988), cognitive (Erblich and Earleywine, 1999), and hypothalamic-pituitary-adrenal (HPA) system alteration (Schuckit et al., 1988, 1987b) in 
response to an acute alcohol dose, and this turned out to be a risk factor for later development of alcoholism independent from family history for alcoholism (Schuckit and Smith, 1996).

Other authors specifically investigated acute tolerance to alcohol, which was found to be more pronounced in the sons and daughters of alcoholics (Blekher et al., 2002; Morzorati et al., 2002). More of this literature was reviewed earlier by Newlin and Thomson (1990).

While these results imply that developing less of the intoxicating alcohol effects can promote alcoholism, research on the stress-dampening properties of alcohol suggests that those may actually be more pronounced in offspring of alcoholics. In the context of hypotheses referring to alcohol-induced tension-reduction (Conger, 1956) or appraisal disruption (Sayette, 1993), this might comprise another mechanism of risk. The autonomic and physiological responses to stressors such as electrical shocks are dampened by prior alcohol administration and this effect is stronger in offspring of alcoholics or even limited to this population (Sinha et al., 1998; Conrod et al., 1998; Finn et al., 1992, 1990; Finn and Pihl, 1987; Sher and Levenson, 1982). Some more recent studies looked at the core parameter defining a stress response, i.e., activation of the hypothalamic-pituitary- adrenal (HPA) system and found somewhat conflicting results: both Uhart et al. (2006) and Zimmermann et al. (2004a,b) found that the HPA response to public speaking stress was higher in offspring of alcoholics than in family history negative controls, but was brought down to the controls' level by prior administration of a moderate alcohol dose. Using a different stressor, Dai et al. observed less adrenocorticotropin (ACTH) response in high-risk than lowrisk subjects, which was equally dampened in both risk groups (Dai et al., 2002). Possible explanations for this inconsistency were discussed earlier (Zimmermann et al., 2004a). They include that, in contrast to our study, Dai et al. used a mere cognitive stressor without psychosocial component, which resulted in considerably lower endocrine stress response. Also, their definition of genetic risk required alcoholism in the father and grandfather, and part of the risk group differences observed by them was due to baseline differences in ACTH secretion.

In addition to the HPA system, several other hormones are activated in response to acute stress, including prolactin (Van de Kar and Blair, 1999). While the functional role of prolactin in the stress response remains unclear, this hormone might be particularly interesting for addiction research for two reasons: (i) its unique regulation by dopamine (Ben Jonathan and Hnasko, 2001); (ii) a prior report of less prolactin stimulation in response to a high-dose alcohol challenge in sons of alcoholics (Schuckit et al., 1987a). Prolactin secretion is controlled by the dopaminergic tuberoinfundibular system, consisting of neurons located in the hypothalamic arcuate and paraventricular nuclei that project to the median eminence. Dopamine secreted by these neurons reaches the anterior pituitary via the long portal vessels where it binds to dopamine D2 receptors on lactotroph cells, resulting in robust suppression of prolactin release (Ben Jonathan and Hnasko, 2001). Therefore, medial basal hypothalamic ablation (Sato et al., 1996) or treatment with dopamine receptor antagonists results in tonically increased prolactin levels.

Stimulatory control of prolactin is more complex and involves heterogeneous factors, some of which act at least partly via inhibition of hypothalamic dopaminergic neurons. This applies to endogenous opiates and to a lesser extent to serotonin and GABA, while vasoactive intestinal peptide and thyrotropin-releasing hormone stimulate prolactin directly at the level of the pituitary. In general, the stimulant effect of prolactin-releasing factors is considerably less potent than dopaminergic inhibition; therefore, most researchers agree that inhibition by dopamine represents the main mechanism controlling prolactin release (Ben Jonathan and Hnasko, 2001). 
Several researchers found that alcohol administration stimulated prolactin secretion in rats (Sato et al., 1996) and in humans. After doses ranging up to $0.8 \mathrm{~g} / \mathrm{kg}$, this effect was equal between groups with vs. without a paternal history for alcoholism (PHA) (Schuckit et al., 1987a; Moss et al., 1989). However, after administration of $0.9 \mathrm{~g} / \mathrm{kg}$, Schuckit et al. (1987a) observed less prolactin stimulation in the PHA group. The recent family history study by Uhart et al. (2006) found no risk group differences in prolactin response to public speaking stress, but did not include an alcohol challenge.

For these reasons we investigated whether the prolactin response to alcohol challenge, public speaking stress, and their combination differs between sons of alcoholics compared to family history negative controls.

Prolactin was measured in samples obtained during a study that was described earlier and consisted of four experimental sessions (Zimmermann et al., 2004a,c). The first part (first 2 days) involved psychosocial stress exposure. During the second part (days 3 and 4), acoustic startle response was studied which did neither involve psychosocial stress nor activate the HPA system. Therefore the second part served as a no-stress comparison condition. Both paradigms involved prior randomized administration of alcohol $(0.6 \mathrm{~g} / \mathrm{kg})$ or placebo. Together, these four experiments allowed us to control for all experimental factors, i.e., genetic risk, stress exposure, and alcohol administration. Based on our prior findings concerning stress-induced HPA stimulation we hypothesized that alcohol would dampen the prolactin stress response and that this effect would be more pronounced in sons of alcoholics than in family history negative (FHN) controls.

\section{Methods}

\subsection{Subjects}

Recruitment of subjects was based on a prospective longitudinal epidemiologic study involving a representative sample of Munich area adolescents (Lieb et al., 2000), which implied that all subjects were between 18 and 27 (median =19) years old. An extensive description of recruitment and the process of verifying inclusion and exclusion criteria is given in a prior report (Zimmermann et al., 2004a) and summarized in the accompanying supplementary material. Fifty-one males were recruited for participation on the basis of their parents' confirmation of a paternal lifetime diagnosis of alcohol dependence following administration of the M-CIDI (Lachner et al., 1998). Accordingly, 45 FHN subjects were recruited to participate after M-CIDI interviews confirmed the absence of any life-time DSMIV alcohol-related disorder in both parents.

All parents were also asked whether they had ever suffered from symptoms of psychiatric disorders, namely generalized anxiety disorder, panic attacks, phobias, depression, mania, psychotic disorders, or eating disorders, and whether alcohol had ever caused health or social problems in their parents, brothers or sisters (i.e., the four grandparents and blood-related uncles and aunts of the study participants). FHN subjects were only included in the control group if both parents denied alcohol-related problems in their first-grade relatives. All subjects were of Caucasian ethnicity.

Eligible subjects were invited for a screening visit performed by a psychiatrist. A medical history was taken, and a physical examination, routine laboratory tests, and a psychiatric interview were performed. Current active alcohol or substance use disorders were excluded by asking the respective CIDI questions and by confirming that liver enzymes and mean 
erythrocyte corpuscular volume were within the normal range. All study procedures were explained including announcement of an unspecified „stress test“, and subjects were mislead concerning the occurrence of placebo sessions by misinforming them that they would receive „different amounts“ of alcohol on all test days. They were instructed to abstain from any illegal substance use throughout the study period and from alcohol use for 3 days before each experimental day, and to have a meal at least $1 \mathrm{~h}$ before arrival to the laboratory. Written informed consent was obtained from all subjects. The study protocol was approved by the local ethical committee and performed in accordance with the Declaration of Helsinki. Subjects were paid for participation.

\subsection{Experimental procedures}

Subjects were tested four times with a minimum of 1 week between sessions. We investigated the response to psychosocial stress on the first 2 days and the acoustic startle reflex on the others. Cigarette smoking and food intake was not allowed during experiments. Alcohol or placebo was administered orally in a randomized, double-blind, placebo-controlled crossoverdesign. Randomization was done on the first day by blindly drawing a prepared envelope that determined whether subjects received alcohol on the first and placebo on the second day, or vice versa. On the third day, another envelope was drawn to independently randomize alcohol administration on days 3 and 4 . Subjects drank $0.6 \mathrm{~g} / \mathrm{kg}$ laboratory grade ethanol diluted in ice-cold grapefruit juice to give a concentration of $15 \%(\mathrm{v} / \mathrm{v})$, or the respective volume of plain grapefruit juice. Drinks were consumed as two equal portions, each consumed within 5 min and separated by a 15 min break. Blood alcohol concentration (BAC) was measured at regular intervals using an Alcotest 7410 breath analyzer (Draeger Sicherheitstechnik, Lübeck, Germany). The experimenter was blind against the subjects' risk status and the alcohol content of the beverage.

On the stress days, subjects reported at the laboratory at $1300 \mathrm{~h}$. At $1315 \mathrm{~h}$, an i.v. line was established and $40 \mathrm{ml} / \mathrm{h}$ of $0.9 \%$ saline was infused throughout the session to keep the line patent for blood drawings. Drinking started at $1400 \mathrm{~h}$. At $1435 \mathrm{~h}$ the subjects were instructed about the test procedures and were given 10 min to prepare an oral presentation as for a job interview. At $1450 \mathrm{~h}$, three health care professionals walked into the room to act as an audience, and the stress test was performed by having the subjects deliver a 5 min selfdisclosing speech during which they tried to convince the audience to give them a job. Thereafter, they were asked to perform mental arithmetics and were immediately prompted for mistakes by the audience (Kirschbaum et al., 1993). Immediately after the stress test, subjects rated how stressful the test had been for them.

On the non-stress days, acoustic startle experiments were performed. Subjects arrived at the same laboratory at $1630 \mathrm{~h}$ and drinking started at 1725 . Four consecutive startle experiments with different methods of startle reflex modulation were performed on each day, which implied plain startle (beginning 35 min after drinking onset), prepulse inhibition (at $40 \mathrm{~min}$ ), fear potentiation (at $50 \mathrm{~min}$ ), and emotional modulation (at $65 \mathrm{~min}$ ). Details were described in a recent paper (Zimmermann et al., 2004c). Startle stimuli were $50 \mathrm{~ms}$ bouts of white noise at $103 \mathrm{~dB}(\mathrm{~A})$ over a background white noise of $79 \mathrm{~dB}(\mathrm{~A})$, presented binaurally via headphones. These procedures did not stimulate the HPA system (Zimmermann, unpublished data). Blood samples during all four experiments were drawn at the times indicated in Fig. 1. 


\subsection{Sample treatment and hormone measurements}

Blood samples were drawn into devices pre-treated with EDTA and aprotinin (2000 kallikrein-inhibiting units per $7 \mathrm{ml}$ of blood, Bayer Leverkusen, Germany). The blood was chilled on ice immediately, spun at $1500 \mathrm{x}$ g within $60 \mathrm{~min}$, and the plasma was frozen at -80 ${ }^{\circ} \mathrm{C}$. Stress day samples of one FHN and three PHA participants and startle day samples of one PHA and seven FHN subjects were unavailable for prolactin assay. Prolactin was measured by an electrochemo-luminescence-immunoassay (ECLI, Roche Diagnostics, Mannheim, Germany) with a detection limit of $0.047 \mathrm{ng} / \mathrm{ml}$. The monoclonal antibody did not cross-react with hGH, hCG, hPL, TSH, FSH, or LH. Inter- and intra-assay coefficients of variation (CV) at $14.1 \mathrm{ng} / \mathrm{ml}$ were 4.0 and $5.0 \%$, respectively.

\section{Statistical methods}

The effect of stress exposure and the ensuing endocrine response was expressed as response area under the time curve (rAUC) extending from the last sample obtained before instructing the participant (i.e., sample 3) to the end of the experiment. The rAUC was calculated by trapezoidal approximation to obtain the total AUC, from which the rectangle defined by sample 3 and the total observation time was subtracted to adjust for differences in the baseline hormone level immediately before stress onset (Pruessner et al., 2003). rAUC values could be negative numbers if the stress-induced prolactin increase was smaller than a subsequent decline below baseline levels. rAUCs were submitted to repeated measures ANOVA with respect to the factors ,treatment“ and „risk group“. To control for potentially confounding effects of some of the individuals' characteristics, all variables of Table 1 which differed between risk groups were included as covariates into the model. Significant factor interactions were analyzed post hoc using t-tests for independent, respectively, paired samples. Interrelations of subjective stress perception with endocrine stress response and of prolactin rAUCs with cortisol and ACTH rAUC were tested by Pearson's product-moment correlations.

Prolactin levels during the non-stress experiments were expressed as rAUCs as described above, but using sample 1 for baseline adjustment. The factors „treatment“ and „risk group“ were tested for their influence on prolactin baseline level and rAUC using two-way ANOVAs for repeated measures and post hoc pairwise comparisons. All data were analyzed using SPSS 14.02 .

\section{Results}

Due to the reasons provided in the supplementary material, data from 22 FHN and 26 FHA participants could be analyzed. Their characteristics are provided in Table 1. PHA scored significantly higher than FHN subjects on the sensation seeking scale, had more alcoholic drinks per week, were more often regular smokers, and more often had a parent reporting psychiatric symptoms. Prolactin secretion dynamics throughout the four experiments is depicted in Fig. 1. The number of samples available for prolactin assays was 22 FHN and 26 PHA for the stress experiments and 16 FHN and 26 PHA subjects for non-stress experiments.

\subsection{Stress days}

Mean and S.D. BAC at the time of stress onset was $55 \pm 18 \mathrm{mg} \%$ in FHN and $56 \pm 15 \mathrm{mg} \%$ in PHA subjects. A repeated-measures ANOVA including all 7 BAC readings did not reveal a significant time by risk group interaction $[F(6,41)=1.1$, n.s.]. Baseline prolactin levels before 
stress onset were influenced by a significant interaction of the factors „treatment“ (alcohol vs. placebo) and „time“ [three samples during the period before stress onset, $F(2,92)=4.40 ; \mathrm{p}=$ .006], but not by risk group. Ingestion of placebo, but not alcohol was associated with a slight decrease of pre-stress prolactin [sample 1 vs. 3 in placebo sessions: $t(47)=3.06$; $p=.004$ ], resulting in significantly lower prolactin levels after placebo compared to alcohol in the sample 3, which immediately preceded stress onset [t $(47)=-3.28 ; \mathrm{p}=.002]$.

That the stressor actually did provoke a significant prolactin increase was confirmed by comparing sample 3 to sample 6 in the placebo experiment [effect of time $F(1,46)=55.9$; $p<$ .001 , no significant time by risk group interaction].

The magnitude of the prolactin stress response, expressed as rAUC, was influenced by „treatment“ $[\mathrm{F}(1,46)=4.07 ; \mathrm{p}=.049]$, but not by „risk group“. A significant „treatment“ by „risk group“ interaction $[\mathrm{F}(1,46)=5.76 ; \mathrm{p}=.02$ prompted us to analyze risk group differences during both treatment conditions and treatment effects in both risk groups. These post hoc group comparisons demonstrated a significant risk group difference during alcohol $[\mathrm{t}(46)=$ 2.45; $\mathrm{p}=.018$ ], but not placebo sessions (see Fig. 2). Alcohol drinking significantly reduced reactivity to subsequent stress in PHA [t $(25)=3.56$; $\mathrm{p}=.002]$, but not in FHN participants. We also tested whether „administration sequence“ (first day placebo/second day alcohol vs. vice versa) affected prolactin rAUC by including this third influential factor into the afore described ANOVA model. No significant main or interaction effects involving administration sequence were detectable, and the risk group by treatment interaction remained the only effect significantly influencing prolactin. This result was unaltered when the above described variables distinguishing the risk groups were controlled for by including them as covariates into the ANOVA; namely sensation seeking, smoking status, drinks per week, and parental history of psychiatric symptoms.

No significant associations of subjectively perceived stress level and prolactin stress response were found in correlation analyses performed separately for each risk group during both alcohol and placebo day.

Data on cortisol and ACTH during the stress experiments have been described earlier (Zimmermann et al., 2004a). The alcohol effect on prolactin (i.e., the difference between hormone rAUC on alcohol and placebo days) was moderately related to the alcohol effect on ACTH (Pearsons's $r=.44, p<.01)$ and on cortisol $(r=.53, \mathrm{p}<.01)$, while the correlation between the alcohol effects on ACTH and on cortisol was closer $(r=.71, p<.01)$.

\subsection{Non-stress days}

This highest BAC level was recorded at 45 min after drinking started, and was $63 \pm 14 \mathrm{mg} \%$ in FHN and $69 \pm 15 \mathrm{mg} \%$ in PHA subjects. Repeated-measures ANOVA with all 6 BAC readings did not reveal a significant time by risk group interaction $[F(5,36)=1.7$, n.s.]. The baseline prolactin levels before drinking did not vary significantly depending on „treatment“ or „group“. When prolactin dynamics throughout the experiments were examined, no main effect of family history and no interaction with treatment was found to affect rAUC. Alcohol administration was associated with significantly higher prolactin rAUC compared to placebo $\mathrm{F}(1,40)=11.91 ; \mathrm{p}=.001]$. Prolactin secretion increased immediately following alcohol ingestion, peaked between samples 3 and 4, and thereafter declined continuously. No such increase was visible during the placebo experiments. 


\section{Discussion}

This study confirms prior reports that a challenge with an intermediate dose of ethanol (Schuckit et al., 1987a) and exposure to a psychosocial stress test (Uhart et al., 2006) stimulate prolactin secretion in healthy subjects. It also replicates that these two interventions per se do not reveal differences between subjects with vs. without a family history of alcoholism. New findings from the current study indicate that: (i) the combination of both interventions, i.e., alcohol administration and subsequent stress exposure specifically attenuates the prolactin stress response in PHA, but not in FHN young adult males without substance-related or other psychiatric disorders and (ii) alcohol-induced dampening of the prolactin stress response is positively correlated with that of ACTH and cortisol.

To interpret these findings we will first discuss mechanisms by which stress and alcohol can affect prolactin secretion. Given the potent regulation of prolactin by the tuberoinfundibular dopamine (TIDA) system, the question arises whether the risk group differences observed in this study can be explained by altered TIDA activity. Rodent experiments clearly support that reduced TIDA secretion is a mechanism by which stress can stimulate prolactin, although it is neither the only one nor an obligatory precondition (Van de Kar and Blair, 1999; Gala, 1990). The underlying neuronal pathways can be inferred from experiments in brain-lesioned rodents and include the cortex, basolateral and central nucleus of the amygdala, and the bed nucleus of the stria terminalis (BNST), whose neurons project to the hypothalamus (Van de Kar and Blair, 1999). Here they connect to the arcuate and paraventricular nucleus, i.e., the source of tuberoinfundibular dopamine.

From animal and human studies it is known that ethanol has a moderately stimulant effect on prolactin (Sato et al., 1996; Schuckit et al., 1987a; Moss et al., 1989). This might be explained by the typical alcohol effects of enhancing GABAergic and opiatergic neurotransmission while blocking NMDA receptors, since pharmacological interventions mimicking those effects also result in prolactin stimulation (Willoughby et al., 1986; Seilicovich et al., 1985; Bregonzio et al., 1998). In addition, alcohol was shown to stimulate prolactin directly at the pituitary (Sato et al., 1996). These findings match with our observation in FHN participants, namely that ingesting $0.6 \mathrm{~g} / \mathrm{kg}$ ethanol increased baseline prolactin and did not impede stressinduced prolactin response. The fact that PHA subjects had the same prolactin responses to stress alone and to alcohol alone as did FHN suggests that their TIDA physiology is intact and is not differently modulated by alcohol than it is in FHN controls. Therefore, we believe that the contrasting alcohol effects observed in PHA, i.e., inhibiting stress-induced prolactin while stimulating its baseline secretion, cannot be explained by altered TIDA functioning. As an alternative explanation, we suppose that the higher brain areas described above to control the TIDA response to stress are specifically inhibited by alcohol in PHA but not in FHN subjects.

If the effect of alcohol on those structures is altered, this could easily explain why we observed that alcohol affects not only the prolactin, but also the HPA stress response. In fact, individual alcohol-induced changes of the prolactin stress response were positively correlated with those of ACTH and cortisol. The results reported here also complement earlier reports on risk group differences in stress response of the HPA system, which was found to be attenuated by prior alcohol drinking in offspring of alcohol-dependent parents, but not in FHN controls (Uhart et al., 2006; Zimmermann et al., 2004a,b). When viewed together, these results confirm that alcohol-induced stress response dampening is specific to high-risk subjects. Since it occurs in two neuroendocrine systems which are differently regulated at the level of the hypothalamus, this appears to be a phenomenon that affects the stress response in general. 
One example for a superior neuroendocrine mechanism that can control stress effects on both HPA and prolactin secretion, and possibly even more aspects of the stress response, is the recently discovered prolactin-releasing peptide (PrRP, see Sun et al., 2005 for review).

Despite its namegiving ability to stimulate prolactin release, studies on its localization and physiology showed that PrRP is not a hypophysiotropic hormone. Rather, it is located in the brain stem solitary tract nucleus and is thought to modulate the activity of several specific nuclei of the amygdala, thalamus, and hypothalamus that are involved in generating a stress response. Specifically, PrRP stimulates the HPA system, increases blood pressure, reduces food intake, and increases body temperature. Various forms of experimental stress, including conditioned fear, were shown to increase central nervous PrRP activity. This suggests that PrRP is an important mediator of stress response and might represent a molecular mechanism responsible for the parallel attenuation of prolactin, ACTH, vasopressin and cortisol response that characterized PHA subjects who drank alcohol before undergoing stress exposure.

Several limitations of our study should be kept in mind. A major limitation is that we tested only male offspring of male alcoholics, which is known to be associated with a strong genetic effect but does not necessarily tell about inheritance to daughters or from mothers (Pollock et al., 1987).We did so to circumvent the methodological difficulties ensuing from menstrual cycle changes in a repeated-measures design. Another weakness is that, for legal reasons, we could not test subjects before age 18. As a consequence, the two risk groups already differed in their current intake of alcohol, in smoking, and in other baseline measures described in Table 1 which is in line with previous findings in offspring of alcoholics (Newlin and Thomson, 1990). Therefore, our results might have been biased by a chronic influence of alcohol or smoking. Since the mentioned characteristics had no significant effect, however, when included into the ANOVA models analyzing effects on the stress response, we think that they actually did not influence our main result of increased alcohol dampening of the stress response in FHP vs. FHN subjects. With respect to smoking, this assumption is further supported by a paper reporting that nicotine administration does not alter early afternoon prolactin secretion (Pickworth et al., 1998).

Comparison of the stress vs. non-stress condition in our study is weakened by the fact that these experiments started at different times of the day, i.e., 1400 vs. 1725 h. We decided for this time schedule based on one prior study on circadian rhythmicity of prolactin secretion, which found a clear sleep-independent evening rise of prolactin in women. In men, however, it was much less pronounced and accounted for a mean increase of $2 \mathrm{ng} / \mathrm{ml}$ in plasma prolactin between 1400 and $1800 \mathrm{~h}$ (Waldstreicher et al., 1996). Based on this small circadian variability of prolactin secretion in men, we decided to value laboratory utilization higher and to schedule two experiments per day in different subjects. Steady baseline secretion, however, does not entirely rule out the possibility of time-dependent differences in prolactin response to external stimuli. For example, at first glance in Fig. 1 it appears that the immediate prolactin stimulation by alcohol (i.e., the first three samples) might have been lower during the stress compared to the startle sessions. On the other hand, prolactin tended to decrease immediately after placebo drinking during stress, but not during startle days. Those differences may either be due to circadian changes, or to the fact that the stress sessions always preceded the startle sessions. During the first two sessions, nonspecific procedures such as needle prick and exposure to the laboratory environment might have been more stressful than during the last ones, and therefore might have stimulated prolactin even before we obtained the first blood sample. Correcting for placebo, the immediate response to alcohol did not significantly differ between stress and startle days. 
A specific strength of our study is that our samplewas based on a representative survey randomly selected fromthe general population, which avoids the response bias usually associated with recruitment of participants by public announcements. This approach prevented us from specifically recruiting subjects with a multigenerational history of alcoholism. Rather, we increased the between-group contrast in genetic risk by excluding all subjects with alcohol problems in second-degree relatives from the control group. By chance, 10 of the 26 PHA subjects had at least one grandparent with alcohol problems.A second strength is that we thoroughly scrutinized the family history of substance-use disorders by direct interviews with both parents concerning themselves and their first-grade relatives, rather than asking the subjects about their parents, grandparents, uncles and aunts.

Concluding from peripheral endocrine findings on underlying central nervous processes is notoriously difficult. Nevertheless, the results reported here confirm the well-known stressdampening effect of alcohol in high-risk subjects and strongly suggest that it is brought about by alcohol actions on brain circuits hierarchically located above the level of the hypothalamus. This view implies that future research on genetic and physiological mechanisms underlying the risk conveyed by gene-stress-alcohol interactions might be most promising when directed to higher brain functions that orchestrate the autonomic, emotional and cognitive aspects of homeostasis during stress exposure.

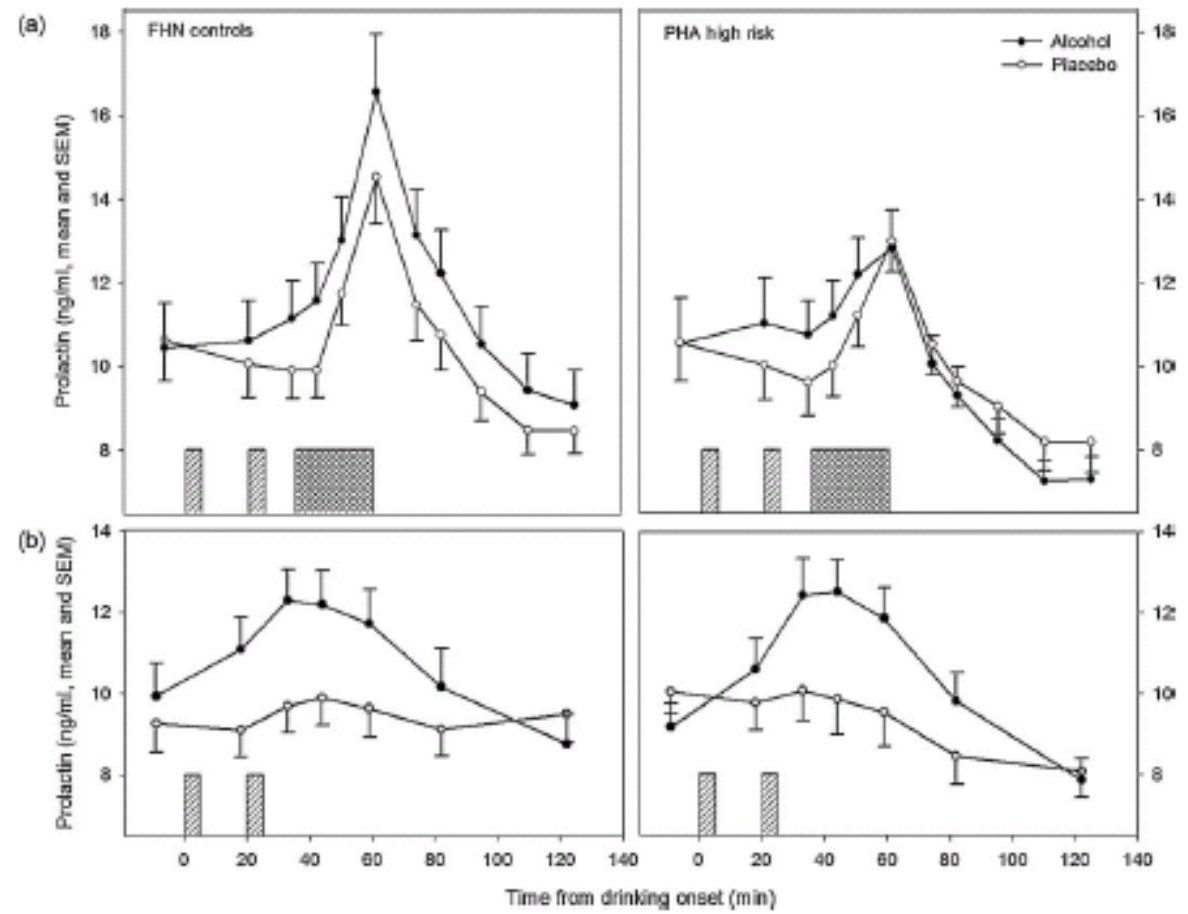

Figure 1 Prolactin secretion during stress (upper panels) and non-stress experiments (i.e., acoustic startle; lower panels) in control (FHN) and high-risk (PHA) groups. Hatched bars denote drinking periods. Duration of stress exposure is highlighted in gray, including preparation time (10 min), job interview, and mental arithmetics (each $5 \mathrm{~min}$ ). Time zero corresponds to $1400 \mathrm{~h}$ for the stress and $1725 \mathrm{~h}$ for the non-stress days. 


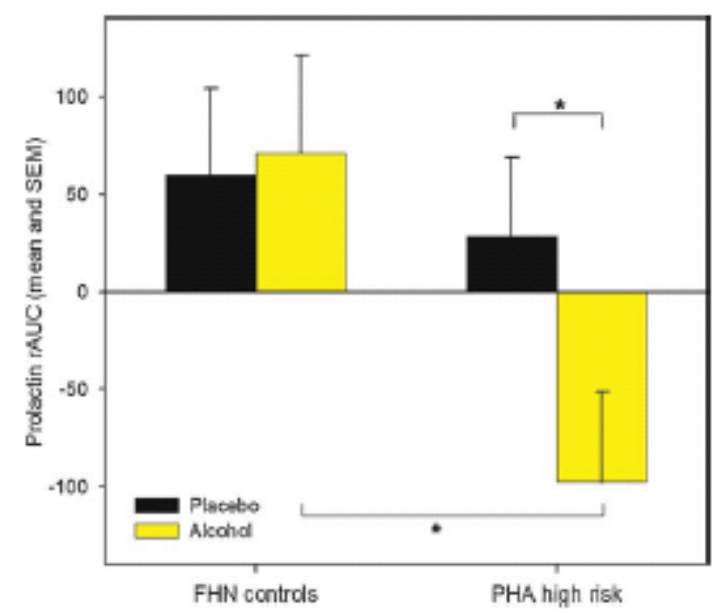

Figure 2 Prolactin response rAUCs for the stress experiments in controls (FHN, $n=22$ ) and high-risk participants (PHA, $n=26$ ). $* p<.05$ in t-tests.

\section{Table 1 Participants' characteristics.}

\begin{tabular}{|c|c|c|c|}
\hline & FHN $(n=22)$ & PHA $(n=26)$ & Test statistic \\
\hline Age (years) & $20.5 \pm 2.3$ & $19.8 \pm 2.7$ & $U=203, p=.08$ \\
\hline Beck depression inventory & $3.5 \pm 6.2$ & $4.0 \pm 4.4$ & $t=-0.35, p=.72$ \\
\hline Sensation seeking scale & $20.4 \pm 6.8$ & $24.2 \pm 5.6$ & $t=-2.10, p=.0$ \\
\hline Drinks per week & $4.7 \pm 4.1$ & $9.4 \pm 7.7$ & $U=168, p=.01$ \\
\hline State anxiety on stress-placebo day & $1.9 \pm 3.7$ & $2.2 \pm 3.0$ & $t=-0.63, p=.50$ \\
\hline State anxiety on stress-alcohol day & $1.4 \pm 2.8$ & $1.9 \pm 2.7$ & $t=-1.00, p=.32$ \\
\hline Cigarettes a lready smoked on stress-placebo day & $1.9 \pm 3.7$ & $2.2 \pm 3.0$ & $U=224, p=.25$ \\
\hline Cigarettes a lready smoked on stress-alcohol day & $1.4 \pm 2.8$ & $1.9 \pm 2.7$ & $U=213.5, p=.19$ \\
\hline Regular smokers (\%) & $7(32)$ & $16(62)$ & $x^{2}=6.70 p=.01$ \\
\hline Used illegal drugs during past 6 months $(\%)$ & $6(27)$ & $12(46)$ & $x^{2}=1.81 p=.15$ \\
\hline Prior history of alcohol use disorder $(\%)$ & $7(32)$ & $11(42)$ & $x^{2}=0.14 p=.48$ \\
\hline Individual history of affective, anxiety, or somatoform disorder $(\%)$ & $2(9)$ & $3(12)$ & \\
\hline Parental history of psychiatric symptoms (\%) & $7(32)$ & $18(69)$ & $x^{2}=6.68 p=.01$ \\
\hline
\end{tabular}

Numbers represent mean \pm S. D. or number and percentage of cases, respectively. Subjects wereeither family history negative (FHN) or had a paternal history of alcoholism (PHA). $t$-Tests were only used for normally distributed variables.

\section{Role of funding source}

This work was supported by the Deutsche Forschungsgemeinschaft (DFG) grant LA 1148/1-1 and by the Max- Planck-Society. Both institutions had no further role in the study design, in the collection, analysis and interpretation of data; in the writing of the report; and in the decision to submit the paper for publication.

\section{Conflict of interest}

H.-U. Wittchen has received grants formPfizer, Lilly, Novartis and Sanofi-Aventis, is on Advisory Boards of Pfizer, Organon, and Wyeth and has received lecture fees of these companies. All other authors declare that they have no conflict of interest.

\section{Acknowledgements}

This work was supported by the Deutsche Forschungsgemeinschaft (DFG) grant LA 1148/1-1 and by the Max- Planck-Society.

We gratefully acknowledge expert technical support by Sabine Rickel. 


\section{Appendix A. Supplementary data}

Supplementary data associated with this article can be found, in the online version, at doi:10.1016/j.psyneuen. 2009.01.015.

\section{References}

- Ben Jonathan, N., Hnasko, R., 2001. Dopamine as a prolactin (PRL) inhibitor. Endocr. Rev. 22, 724-763.

- Blekher, T., Ramchandani, V.A., Flury, L., Foroud, T., Kareken, D., Yee, R.D., Li, T.K., O’Connor, S., 2002. Saccadic eyemovements are associated with a family history of alcoholism at baseline and after exposure to alcohol. Alcohol. Clin. Exp. Res. 26, 15681573.

- Bregonzio, C., Navarro, C.E., Donoso, A.O., 1998. NMDA receptor antagonists block stressinduced prolactin release in female rats at estrus. Eur. J. Pharmacol. 350, 259—265.

- Conger, J.J., 1956. Reinforcement theory and the dynamics of alcoholism. Quart. J. Stud. Alcohol 17, 296-305.

- Conrod, P., Pihl, R.O., Vassileva, J., 1998. Differential sensitivity to alcohol reinforcement in groups of men at risk for distinct alcoholism subtypes. Alcohol. Clin. Exp. Res. 22, 585-597.

- Dai, X., Thavundayil, J., Gianoulakis, C., 2002. Response of the hypothalamic-pituitaryadrenal axis to stress in the absence and presence of ethanol in subjects at high and low risk of alcoholism. Neuropsychopharmacology 27, 442-452.

- Erblich, J., Earleywine, M., 1999. Children of alcoholics exhibit attenuated cognitive impairment during an ethanol challenge. Alcohol. Clin. Exp. Res. 23, 476-482.

- $\quad$ Finn, P.R., Earleywine, M., Pihl, R.O., 1992. Sensation seeking, stress reactivity, and alcohol dampening discriminate the density of a family history of alcoholism. Alcohol. Clin. Exp. Res. 16, 585-590.

- $\quad$ Finn, P.R., Pihl, R.O., 1987. Men at high risk for alcoholism: the effect of alcohol on cardiovascular response to unavoidable shock. J. Abnorm. Psychol. 96, 230—236.

- Finn, P.R., Zeitouni, N.C., Pihl, R.O., 1990. Effects of alcohol on psychophysiological hyperreactivity to nonaversive and aversive stimuli in men at high risk for alcoholism. J. Abnorm. Psychol. 99,79—85.

- Gala, R.R., 1990. The physiology and mechanisms of the stressinduced changes in prolactin secretion in the rat. Life Sci. 46,1407-1420.

- Hada, M., Porjesz, B., Chorlian, D.B., Begleiter, H., Polich, J., 2001. Auditory P3a deficits in male subjects at high risk for alcoholism. Biol. Psychiatry 49, 726-738.

- Heath, A.C., Bucholz, K., Madden, P.A., Dinwiddie, S.H., Slutske, W.S., Bierut, L., Statham, D.J., Dunne, M.P., Whitfield, J.B., Martin, N.G., 1997. Genetic and environmental contributions to alcohol dependence risk in a national twin sample: consistency of findings in women and men. Psychol. Med. 1381-1396.

- Hill, S.Y., De, B.M., Keshavan, M.S., Lowers, L., Shen, S., Hall, J., Pitts, T., 2001. Right amygdala volume in adolescent and young adult offspring from families at high risk for developing alcoholism. Biol. Psychiatry 49, 894-905.

- Kirschbaum, C., Pirke, K.M., Hellhammer, D.H., 1993. The 'Trier social stress test' - a tool for investigating psychobiological stress responses in a laboratory setting. Neuropsychobiology 28, 76-81.

- $\quad$ Lachner, G., Wittchen, H.U., Perkonigg, A., Holly, A., Schuster, P., Wunderlich, U., Turk, D., Garczynski, E., Pfister, H., 1998. Structure, content and reliability of the Munich-Composite International Diagnostic Interview (M-CIDI) substance use sections. European Addiction Research 4, 28-41. 
- Lieb, R., Isensee, B., von Sydow, K., Wittchen, H.U., 2000. The early developmental stages of psychopathology study (EDSP): a methodological update. Eur. Addic. Res. 6, 170—182.

- Morzorati, S.L., Ramchandani, V.A., Flury, L., Li, T.K., O’Connor, S., 2002. Self-reported subjective perception of intoxication reflects family history of alcoholism when breath alcohol levels are constant. Alcohol. Clin. Exp. Res. 26, 1299-1306.

- Moss, H.B., Yao, J.K., Maddock, J.M., 1989. Responses by sons of alcoholic fathers to alcoholic and placebo drinks: perceived mood, intoxication, and plasma prolactin. Alcohol. Clin. Exp Res. 13, 252-257.

- Newlin, D.B., Thomson, J.B., 1990. Alcohol challenge with sons of alcoholics: a critical review and analysis. Psychol. Bull. 180, 383-402.

- Pickworth, W.B., Fant, R.V., 1998. Endocrine effects of nicotine administration, tobacco and other drug withdrawal in humans. Psychoneuroendocrinology 23, 131-141.

- $\quad$ Pollock, V.E., Schneider, L.S., Garbrielli, W.F., Goodwin, D.W., 1987. Sex of parent and offspring in the transmission of alcoholism. A meta-analysis. J. Nerv. Ment. Dis. 175, 668672.

- $\quad$ Prescott, C.A., Kendler, K.S., 1999. Genetic and environmental contributions to alcohol abuse and dependence in a population-based sample of male twins. Am. J. Psychiatry 156, 34—40.

- $\quad$ Pruessner, J.C., Kirschbaum, C., Meinlschmid, G., Hellhammer, D.H., 2003. Two formulas for computation of the area under the curve represent measures of total hormone concentration versus timedependent change. Psychoneuroendocrinology 28, 916-931.

- Sato, F., Nakamura, K., Taguchi, M., Aoki, H., Aoki, T., Yasuda, N., 1996. Studies on the site of ethanol action in inducing prolactin release in male rats. Metab.: Clin. Exp. 45, 13301334.

- Sayette, M.A., 1993. An appraisal-disruption model of alcohol's effects on stress responses in social drinkers. Psychol. Bull. 114, 459—476.

- Schuckit, M.A., Golba, K., Risch, C., 1987a. Serum prolactin levels in sons of alcoholics and control subjects. Am. J. Psychiatry 144, 854-859.

- Schuckit, M.A., Gold, E., Risch, C., 1987b. Plasma cortisol levels following ethanol in sons of alcoholics and controls. Arch. Gen. Psychiatry 44, 942-945.

- Schuckit, M.A., Gold, E.O., 1988. A simultaneous evaluation of multiple markers of ethanol/placebo challenges in sons of alcoholics and controls. Arch. Gen. Psychiatry 45, $211-216$.

- Schuckit, M.A., Risch, S.C., Gold, E., 1988. Alcohol consumption, ACTH level, and family history of alcoholism. Am. J. Psychiatry 145, 1391-1395.

- Schuckit, M.A., Smith, T.L., 1996. An 8-year follow-up of 450 sons of alcoholic and control subjects. Arch. Gen. Psychiatry 53, 202-210.

- Seilicovich, A., Rubio, M., Duvilanski, B., Munoz, M., Rettori, V.V., 1985. Inhibition by naloxone of the rise in hypothalamic dopamine and serum prolactin induced by ethanol. Psychopharmacology (Berl) 87, 461-463.

- Sher, K.J., Levenson, R.W., 1982. Risk for alcoholism and individual differences in the stress - response-dampening effect of alcohol. J. Abnorm. Psychol. 91, 350-367.

- Sinha, R., Robinson, J., O’Malley, S., 1998. Stress response dampening: effects of gender and family history of alcoholism and anxiety disorders. Psychopharmacology 137, 311-320.

- Sun, B., Fujiwara, K., Adachi, S., Inoue, K., 2005. Physiological roles of prolactin-releasing peptide. Regul. Pept. 126, 27-33.

- $\quad$ Uhart, M., Oswald, L., McCaul, M.E., Chong, R., Wand, G.S., 2006. Hormonal responses to psychological stress and family history of alcoholism. Neuropsychopharmacology 31, 22552263.

- Van de Kar, L.D., Blair, M.L., 1999. Forebrain pathways mediating stress-induced hormone secretion. Front. Neuroendocrinol. 20, 1—48.

- Waldstreicher, J., Duffy, J.F., Brown, E.N., Rogacz, S., Allan, J.S., Czeisler, C.A., 1996. Gender differences in the temporal organization of proclactin (PRL) secretion: evidence for a sleep-independent circadian rhythm of circulating PRL levels_- a clinical research center study. J. Clin. Endocrinol. Metab. 81, 1483-1487. 
- Wand, G.S., Mangold, D., El Deiry, S., McCaul, M.E., Hoover, D., 1998. Family history of alcoholism and hypothalamic opioidergic activity. Arch. Gen. Psychiatry 55, 1114-1119.

- Willoughby, J.O., Jervois, P.M., Menadue, M.F., Blessing,W.W., 1986. Activation of GABA receptors in the hypothalamus stimulates secretion of growth hormone and prolactin. Brain Res. 374,119-125.

- Zimmermann, U., Spring, K., Kunz-Ebrecht, S., Uhr, M., Wittchen, H.U., Holsboer, F., 2004a. Effect of ethanol on hypothalamicpituitary-adrenal system response to psychosocial stress in sons of alcohol-dependent fathers. Neuropsychopharmacology 29, 1156-1165.

- Zimmermann, U., Spring, K., Wittchen, H.U., Himmerich, H., Landgraf, R., Uhr, M., Holsboer, F., 2004b. Arginine vasopressin and adrenocorticotropin secretion in response to psychosocial stress is attenuated by ethanol in sons of alcohol-dependent fathers. J. Psychiatr. Res. 38, 385-393.

- Zimmermann, U., Spring, K., Wittchen, H.U., Holsboer, F., 2004c. Effects of ethanol administration and induction of anxietyrelated affective states on the acoustic startle reflex in sons of alcohol-dependent fathers. Alcohol. Clin. Exp. Res. 28, 424—432. 